\title{
Schwinger, Pegg and Barnett approaches and a relationship between angular and Cartesian quantum descriptions II: Phase Spaces.
}

\author{
M. Ruzzi \\ mruzzi@fsc.ufsc.br - Depto.de Física \\ Universidade Federal de Santa Catarina \\ 88040 - 900 Florianópolis, S.C., Brazil \\ D. Galetti \\ Instituto de Física Teórica \\ Universidade Estadual Paulista \\ Rua Pamplona 145 \\ 01405 - 900 São Paulo, S.P., Brazil
}

\begin{abstract}
Following the discussion - in state space language - presented in a preceding paper, we work on the passage from the phase space description of a degree of freedom described by a finite number of states (without classical counterpart) to one described by an infinite (and continuously labeled) number of states. With that it is possible to relate an original Schwinger idea to the Pegg and Barnett approach to the phase problem. In phase space language, this discussion shows that one can obtain the Weyl-Wigner formalism, for both Cartesian and angular coordinates, as limiting elements of the discrete phase space formalism.

PACS: 03.65.-w, 03.65.Bz, 03.65.Ca
\end{abstract}

\section{INTRODUCTION}

In a previous work it has been shown that the usual quantum descriptions of Cartesian and angular coordinates in state space can both be seen as different limiting cases of the Schwinger program of treating quantum discrete variables [1]. The limiting process involved reproduces the Pegg and Barnett approach to phase variables in the case of angle/angular momentum variables [2]. The purpose of this work is to translate that discussion into a phase space point of view, which might be a way to unify under a same structure three apparently different formalisms, each one adapted to one specific kind of quantum variable, namely Cartesian, angular and discrete. In doing so, we again relate the Pegg and Barnett and Schwinger approaches, now through the phase space representatives of number and phase operators.

The phase-space picture is a well established picture of quantum mechanics [3 10], specially if one deals with degrees of freedom with classical counterpart, a situation in which the Weyl-Wigner formalism is the undisputed approach. Nevertheless, to cope with variables such as rotation angle and angular momentum, the formalism had to be adapted in order to account for the inherent periodicity involved. This was accomplished in the late seventies [11.12] and developed to full extent in 13. However, when one deals with degrees of freedom without classical counterpart, the formulations discussed above are not applicable. In these cases, there is a formalism completely capable to deal with the peculiarities of the finite/discrete character of the variables [14 [16], much in the same spirit of the WeylWigner formalism itself. Following the procedure shown in [1] which led from discrete variables to the cases with classical counterpart, we show that from the discrete phase space formalism naturally emerges the usual Weyl-Wigner formalism and also its rotation angle-angular momentum version. In addition, some properties of the discrete Wigner function are also discussed. From a more rigorous mathematical point of view, these limiting processes presented in this context have been also discussed in Refs. $[17$ 19].

This paper is organized as follows. In section II we briefly present the main ideas of the discrete phase space representation drawing attention to some properties of the discrete Wigner function, while in section III we discuss the limiting processes which lead the original operator bases to the well-known Weyl-Wigner continuous case as well as the particular case of rotations. Finaly, section IV is devoted to the conclusions.

\section{DISCRETE PHASE SPACE}

As has been already shown in the past [14 16], a discrete phase space representation of a quantum mechanical degree of freedom which is characterized by a finite number of states, therefore with no classical counterpart, can 
be established if we are given a basis in the corresponding operator space. One such basis has been introduced by Schwinger in his seminal paper on this subject [20], constructing it out of some particular unitary cyclically shifting pairs of operators, and another one has been proposed that basically considers the double Fourier transform of that of Schwinger [14]. As was previously shown, once we are provided with such an operator basis, it is a direct task to obtain the discrete phase space representatives of the operators acting on the state space from which we started. To briefly summarize those results let us consider the operator basis and recall its main properties.

The discrete phase space formalism is set over the basis elements

$$
G(j, l)=\frac{1}{N} \sum_{m, n=-h}^{h} U^{m} V^{n} \exp \left(\frac{i \pi m n}{N}\right) \exp \left[-\frac{2 \pi i}{N}(m j+n l)\right] \exp [i \pi \phi(m+h, n+h ; N)],
$$

where $(j, l) \in[-h, h], h=\frac{N-1}{2}$ (for simplicity, odd $N^{\prime}$ s will be considered, as even values only require only a little more care and a heavier notation). The modular phase $\phi(m, n ; N)$, included to warrant an explicit mod $N$ symmetry in the summing indices of the basis, is given by

$$
\phi(m, n ; N)=N I_{m}^{N} I_{n}^{N}-m I_{n}^{N}-n I_{m}^{N}
$$

with

$$
I_{k}^{N}=\left[\frac{k}{N}\right]
$$

standing for the integral part of $k$ with respect to $N$. The $U$ 's and $V$ "s are the Schwinger unitary operators [20], shortly reviewed in [1].

As a basis, the set (11) can be used to represent all linear operators acting on the given $N$-dimensional state space; this can be accomplished by a direct decomposition

$$
\hat{O}=\sum_{m, n=0}^{N-1} O(m, n) G(m, n),
$$

where the coefficient, $O(m, n)$, that gives rise to the representative of the operator $\hat{O}$ in the discrete phase space [14], is given by

$$
O(m, n)=\frac{1}{N} \operatorname{Tr}[G(m, n) \hat{O}]
$$

where we used the fact that $G(m, n)$ is self adjoint.

The basic properties of the basis, Eq. (11), are

$$
\begin{gathered}
1) \operatorname{Tr}[G(m, n)]=1 \\
\text { 2) } \operatorname{Tr}\left[G^{\dagger}(m, n) G(r, s)\right]=N \delta_{m, r}^{[N]} \delta_{n, s}^{[N]} ; \\
\text { 3) } \operatorname{Tr}\left[G^{\dagger}(m, n) G(u, v) G(r, s)\right]=\sum_{a, b, c, d=-h}^{h} \frac{1}{N^{2}} \exp \left[\frac{i \pi}{N}(b c-a d)\right] \\
e^{[-i \pi \phi(a+c+h, b+d+h ; N)]} \exp \left\{\frac{2 \pi i}{N}[a(m-u)+b(n-v)+c(m-r)+d(n-s)]\right\},
\end{gathered}
$$

where the last expression is important for the mapping of products of operators [21]. Particular interest resides in the mapping of the commutator of two operators, for then it is possible to study, for example, the time evolution of the density operator in the von Neumann-Liouville equation [16]22]. 


\section{A. The Discrete Wigner function}

The phase space representative of the density operator in the discrete approach is also referred to as (discrete) Wigner function [14,23,24]. If the (pure) state of a given system is described by

$$
|\psi\rangle=\sum_{n} \psi_{n}\left|u_{n}\right\rangle
$$

where $\left\{\left|u_{n}\right\rangle\right\}$ is the (complete and orthonormal) set of eigenvectors of the Schwinger operator $U$, then the use of eq.(5) leads to a Wigner function of the form

$$
\rho_{w}(m, n)=\frac{1}{N^{2}} \sum_{j, l, k} \psi_{k}^{*} \psi_{k-l} \exp \left[\frac{2 \pi i}{N}\left(j k-\frac{j l}{2}-m j-n l\right)\right],
$$

or

$$
\rho_{w}(m, n)=\frac{1}{N^{2}} \sum_{l, k} \psi_{k}^{*} \psi_{k-l} \frac{\sin [\pi(k-m-l / 2)]}{\sin \left[\frac{\pi}{N}(k-m-l / 2)\right]} \exp \left[-\frac{2 \pi i}{N} n l\right] .
$$

Its main properties are, in direct analogy with the usual continuous Wigner function:

1) It is a real function, as it follows from the hermicity of the basis elements.

2) Summing it over each one of its indices gives the probability distribution in the other. For example:

$$
\sum_{n} \rho_{w}(m, n)=\sum_{n} \frac{1}{N^{2}} \sum_{j, l, k} \psi_{k}^{*} \psi_{k-l} \exp \left[\frac{2 \pi i}{N}\left(j k-\frac{j l}{2}-m j-n l\right)\right]
$$

such that

$$
\sum_{n} \rho_{w}(m, n)=\frac{1}{N} \sum_{j, l, k} \psi_{k}^{*} \psi_{k-l} \exp \left[\frac{2 \pi i}{N}\left(j k-\frac{j l}{2}-m j\right)\right] \delta_{l, 0}^{[N]}
$$

and so

$$
\sum_{n} \rho_{w}(m, n)=\left|\psi_{m}\right|^{2}
$$

And in the same way, the summation over $\{m\}$ would lead to the probability distribution associated to the eigenstates of the Schwinger operator $V$.

3) It must be different from zero in at least $N$ sites in the discrete phase space. Writing it as

$$
\rho_{w}(m, n)=\frac{1}{N^{2}} \sum_{j, l} \exp \left[-\frac{2 \pi i}{N}(m j+n l)\right] \sum_{k} \psi_{k}^{*} \psi_{k-l} \exp \left[\frac{2 \pi i}{N}\left(j k-\frac{j l}{2}\right)\right],
$$

it is clear that it is the double Fourier transform of the quantity $\rho_{s}(j, l)$

$$
\rho_{s}(j, l)=\sum_{k} \psi_{k}^{*} \psi_{k-l} \exp \left[\frac{2 \pi i}{N}\left(j k-\frac{j l}{2}\right)\right]
$$

which, by its turn, can be seen as the inner product of two vectors $\left\{\psi_{k} \exp \left[-\frac{2 \pi i}{N} j k\right]\right\}$ and $\left\{\psi_{k-l} \exp \left[-\frac{\pi i}{N} j l\right]\right\}$ of unity length. By the Schwarz inequality it is clear than that $\left|\rho_{s}(j, l)\right|^{2} \leq 1$, and from properties of the discrete Fourier transform, one can also conclude that

$$
\left(\rho_{w}(m, n)\right)^{2} \leq 1
$$


Now, using the property 16

$$
\operatorname{Tr}\left[\hat{O}_{1} \hat{O}_{2}\right]=\frac{1}{N} \sum_{m, n} O_{1}(m, n) O_{2}(m, n)
$$

then

$$
\operatorname{Tr}\left[(|\psi\rangle\langle\psi|)^{2}\right]=\frac{1}{N} \sum_{m, n}\left(\rho_{w}(m, n)\right)^{2},
$$

which leads to

$$
1=\frac{1}{N} \sum_{m, n}\left(\rho_{w}(m, n)\right)^{2}
$$

and considering inequality (16) we conclude that the discrete Wigner function must be different from zero in at least on $N$ sites in the discrete phase space.

\section{THE CONTINUUM LIMIT IN PHASE SPACE}

The continuum limit of an operator representative in phase space is to be seen as its behaviour in the infinite dimensional/continuum limit. We now follow a procedure similar to that of [1].

\section{A. Cartesian coordinates}

We start from the discrete space operator basis elements,

$$
G(j, l)=\frac{1}{N} \sum_{m, n=-h}^{h} U^{m} V^{n} \exp \left(\frac{i \pi m n}{N}\right) \exp \left[-\frac{2 \pi i}{N}(m j+n l)\right],
$$

were we omit the modular phase since we will restrict ourselves to sums in the interval $[-h, h]$. Then we introduce the scaling parameter

$$
\epsilon=\sqrt{\frac{2 \pi}{N}}
$$

which will become infinitesimal as $N \rightarrow \infty$. We also introduce two Hermitian operators $\{P, Q\}$,

$$
P=\sum_{j=-\frac{N-1}{2}}^{\frac{N-1}{2}} j \epsilon^{\delta} p_{0}\left|v_{j}\right\rangle\left\langle v_{j}\left|\quad Q=\sum_{j^{\prime}=-\frac{N-1}{2}}^{\frac{N-1}{2}} j^{\prime} \epsilon^{2-\delta} q_{0}\right| u_{j^{\prime}}\right\rangle\left\langle u_{j^{\prime}}\right|,
$$

constructed out of the projectors of the eigenstates of $U$ and $V$. Again, $\delta$ is a free parameter which might assume any value in the open interval $(0,2) .\left\{p_{0}, q_{0}\right\}$ are real parameters that might carry units of momentum and position, respectively, and $\epsilon^{\delta} p_{0}$ and $\epsilon^{2-\delta} q_{0}$ are the distance between successive eigenvalues of the $P$ and $Q$ operators. With the help of these, we can rewrite the Schwinger operators as

$$
V=\exp \left[\frac{i \epsilon^{2-\delta} P}{p_{0}}\right] \quad U=\exp \left[\frac{i \epsilon^{\delta} Q}{q_{0}}\right]
$$

and perform the change of variables

$$
\begin{aligned}
& q=q_{0} \epsilon^{2-\delta} j \quad p=p_{0} \epsilon^{\delta} l \\
& u=p_{0} \epsilon^{\delta} m \quad v=-q_{0} \epsilon^{2-\delta} n .
\end{aligned}
$$


With that, we arrive at a new operator basis elements that do not explicitely depend on $\delta$, but, at the same time, the operators $U$ and $V$ carry a particular $\epsilon$ dependence, defined by the particular choice of $\delta$, namely

$$
G(p, q)=\frac{1}{q_{0} p_{0} \epsilon^{2} N} \sum_{u, v=-h}^{h} \Delta u \Delta v \exp \left[\frac{i u Q}{p_{0} q_{0}}\right] \exp \left[-\frac{i v P}{p_{0} q_{0}}\right] \exp \left(-\frac{i}{2 p_{0} q_{0}} u v\right) \exp \left[-\frac{i}{p_{0} q_{0}}(q u-p v)\right] .
$$

If we take the limit $N \rightarrow \infty$, it is clear that we can consider $\Delta u \rightarrow d u$ and $\Delta v \rightarrow d v$, yielding

$$
G(p, q)=\frac{1}{2 \pi q_{0} p_{0}} \int_{-\infty}^{\infty} \int_{-\infty}^{\infty} d u d v \exp \left[\frac{i u(Q-q-v / 2)}{p_{0} q_{0}}\right] \exp \left[-\frac{i v(P-p)}{p_{0} q_{0}}\right] .
$$

As we know from [1] that in this limit we recover the usual results for position and momentum once $p_{0} q_{0}=\hbar$, we use the identity

$$
|q\rangle\langle q|=\frac{1}{2 \pi \hbar} \int_{-\infty}^{\infty} d x \exp \left[\frac{i x(Q-q)}{\hbar}\right]
$$

and obtain

$$
\begin{gathered}
G(p, q)=\frac{1}{2 \pi \hbar} \int_{-\infty}^{\infty} d v|q+v / 2\rangle\langle q+v / 2| \exp \left[-\frac{i v(P-p)}{\hbar}\right] \\
G(p, q)=\frac{1}{2 \pi \hbar} \int_{-\infty}^{\infty} d v|q+v / 2\rangle\langle q-v / 2| \exp \left[\frac{i v p}{\hbar}\right],
\end{gathered}
$$

which is exactly the form of the Weyl-Wigner basis elements $\Delta(p, q)$. It is interesting to see that, as in the state space description, the parameter $\delta$ doesn't affect the final result since, in this case (any $\delta \in(0,2)$ ), the basis elements do not depend on it at all, but $U$ and $V$ depend on $\epsilon$. It is now a trivial matter to prove that the decomposition coefficients are well behaved in the limit and also go to the Weyl-Wigner coefficients. From this we see that the whole mapping scheme is recovered. This result was already achieved for the particular case $\delta=1$ in $[16$, where the limiting process which leads to the Moyal bracket was also discussed. Moreover, it has to be stressed that, starting from the continuous family of unitary operators, Eq. (23), and realizing the independence of the basis elements on $\delta$, the Weyl-Wigner basis elements are overdetermined in the limiting process, since, for any $\delta \in(0,2)$ pair of operators, we always get the same final expression. This means that for the continuous family of unitary operators (except for $\delta=0$ or $\delta=2$ ), as proposed, the continuum limit is the Weyl-Wigner operator basis.

From these results one immediately concludes that the discrete Wigner function has the ordinary Wigner function as its continuum limit, in the sense discussed above. As we already stated, most properties of the usual Wigner function are originally present in the discrete one, and come out as the continuum limit of the latter.

In the discrete case we have seen that the Wigner function must be different from zero in at least $N$ sites in phase space. It is obvious that the same procedure which led to this result would lead to the well known property of the usual Wigner function that it must be different from zero in a region of the phase space of area at least $\hbar$. This discussion illustrates somewhat quantitatively how the quantum effects become more and more drastic as the dimensionality $N$ gets smaller.

\section{B. Angular coordinates}

Following on our analogy with what was done in [1], we choose now the parameter $\delta$ in the extreme situation $\delta=0$. We expect now to obtain a phase space formalism which is consistent with angular coordinates. We start again from our discrete operator space basis elements, Eq.(20),

$$
G\left(j^{\prime}, l^{\prime}\right)=\frac{1}{N} \sum_{m^{\prime}, n^{\prime}=-h}^{h} U^{m^{\prime}} V^{n^{\prime}} \exp \left(\frac{i \pi m^{\prime} n^{\prime}}{N}\right) \exp \left[-\frac{2 \pi i}{N}\left(m^{\prime} j^{\prime}+n^{\prime} l^{\prime}\right)\right]
$$

Rewriting the Schwinger operators as above, but with $\delta=0$, we now would have 


$$
M=\sum_{j=-\frac{N-1}{2}}^{\frac{N-1}{2}} j m_{0}\left|v_{j}\right\rangle\left\langle v_{j}\right| \quad \text { and } \quad \Theta=\sum_{j^{\prime}=-\frac{N-1}{2}}^{\frac{N-1}{2}} j^{\prime} \epsilon^{2} \theta_{0}\left|u_{j^{\prime}}\right\rangle\left\langle u_{j^{\prime}}\right| \text {, }
$$

leading to

$$
V=\exp \left[\frac{i \epsilon^{2} M}{m_{0}}\right] \quad \text { and } \quad U=\exp \left[\frac{i \Theta}{\theta_{0}}\right],
$$

so that only $V$ depends now on $\epsilon$, and changing the variables as

$$
\begin{aligned}
\theta & =\theta_{0} \epsilon^{2} j^{\prime} & & l=l_{0} l^{\prime} \\
m & =m_{0} m^{\prime} & & \alpha=-\theta_{0} \epsilon^{2} n^{\prime},
\end{aligned}
$$

we have for the basis elements

$$
\begin{aligned}
G(\alpha, l)= & -\frac{1}{2 \pi \theta_{0}} \sum_{m=-m_{0} h}^{m_{0} h} \sum_{\alpha=\left(\pi-\frac{\pi}{N}\right) \theta_{0}}^{\left(-\pi+\frac{\pi}{N}\right) \theta_{0}} \Delta \theta \exp \left[\frac{i m \Theta}{m_{0} \theta_{0}}\right] \exp \left[-\frac{i \alpha M}{m_{0} \theta_{0}}\right] \\
& \exp \left(-\frac{i m \alpha}{2 m_{0} \theta_{0}}\right) \exp \left[-\frac{i}{m_{0} \theta_{0}}(m \theta-l \alpha)\right] .
\end{aligned}
$$

Performing again the limit $N \rightarrow \infty$, the angle variables become continuous and we have

$$
G(\alpha, l)=\frac{1}{2 \pi \theta_{0}} \sum_{m=-\infty}^{\infty} \int_{-\pi \theta_{0}}^{\pi \theta_{0}} d \alpha \exp \left[i m\left(\Theta-\theta-\frac{\alpha}{2}\right)\right] \exp \left[-\frac{i \alpha(M-l)}{m_{0} \theta_{0}}\right] .
$$

The sum over $m$ is the projector in angle space $\left(\theta_{0}\right.$ is set to 1 , so the angle units are radians, and $m_{0} \theta_{0}$ is set to $\left.\hbar\right)$, and

$$
\left.G(\alpha, l)=\frac{1}{2 \pi} \int_{-\pi}^{\pi} d \alpha|| \theta+\frac{\alpha}{2}\right\rangle\left\langle\theta+\frac{\alpha}{2}\right| \exp \left[-\frac{i \alpha(M-l)}{\hbar}\right] .
$$

so that, with the use Eq.(30), we achieve the result

$$
G(\alpha, l)=\frac{1}{2 \pi} \int_{-\pi}^{\pi} d \alpha\left|\theta+\frac{\alpha}{2}\right\rangle\left\langle\theta-\frac{\alpha}{2}\right| \exp \left[\frac{i l \alpha}{\hbar}\right],
$$

that is precisely the result of references 12.13. We remark that we have no need to worry about the periodicities in the angle variable as our angular states are bounded to the $[-\pi, \pi)$ interval by definition, and our notation has mod $N$ periodicity $(\bmod 2 \pi$ in the continuum limit) by construction $[\mathrm{i}$. It would seem at first glance that the continuum interval is $[-\pi, \pi]$, but that is not the case as it can be seem from the original discrete results that the states in the extremes of the interval are not the same. We understand that, once the basis elements are recovered, the whole mapping procedure is recovered.

Again, all properties of the angular Wigner function can be obtained from its discrete counterpart by the limiting process above. It must be stated however that in a lot of cases it turns out to be easier to work with the discrete rather than in the angular case. That is particularly true in the obtention of the angular counterpart of Eq.(16), which in the angular case doesn't lead to a condition involving a minimal area unit in phase space due to the very nature of the angular phase space.

It is interesting to note that what was considered to be conditions for the existence of the Wigner function in [12, 13 are derived as properties of it in the present scheme.

\section{Mapping of the Pegg and Barnett operators}

The number and phase operators of Pegg and Barnett can be immediately mapped on the discrete phase space. In fact, we exactly reproduce the PB scheme if we rename the $M$ operator of Eq.(31) by $N$ and include a reference angle in the definition of $\Theta$ (which must be an integer multiple of $\frac{2 \pi}{N}$ ). The phase space representatives of these operators, through direct use of Eq.(5), are seen to be

$$
N(m, n)=n, \quad \Theta(m, n)=\theta_{\text {ref }}+\frac{2 \pi}{N} m,
$$

with obvious continuum limits. 


\section{CONCLUSIONS}

Motivated by the results of part I, we looked for a phase space discussion of the limits which connect discrete, angular and Cartesian coordinates. It became then clear that the Weyl-Wigner formalism, in both position-momentum and angle-angular momentum cases, can be seen as limiting elements of a discrete phase space formalism. The angleangular momentum case is seen to be in deep connection with the Pegg and Barnett approach to the phase problem, while the Weyl-Wigner operator basis is reobtained for all the cases for which the parameter governing the unitary operators is different from zero; in this sense the Weyl-Wigner basis is overdetermined in the limiting process. An interesting by-product of this discussion is the analysis of the Wigner function, which reproduced the conditions imposed on the angular Wigner function in [12,13].

With all that in mind, one is compelled to regard this as a kind of standard, or rather 'natural' approach to phase space in quantum mechanics. The basic feature that pertains to all three versions of the formalism is that one constructs a basis in operator space out of the Fourier transform of the shifting operators. A one-to-one correspondence then ensures the existence of a mapping between abstract operators and functions in phase space.

[1] M. Ruzzi, to appear in J. Phys. A: Math and Gen.

[2] D.T. Pegg and S.M. Barnett 1989 Phys. Rev. A39 1665

[3] H Weyl 1978 The Theory of Groups and Quantum Mechanics (Dover, New York)

[4] E. Wigner 1932 Phys. Rev. 40749

[5] J.E. Moyal 1949 Proc. Camb. Phil. Soc. 4599

[6] M. Hillery, R.F. O'Connell, M.O. Scully and E.P. Wigner 1984 Physics Reports C 106121

[7] N.L. Balazs and B.K. Jennings 1984 Physics Reports C 104347

[8] B. Leaf 1968 J. Math. Phys. 965

[9] S. R. de Groot and L.G. Suttorp 1972 Foundations of Electrodynamics (North Holland, Amsterdam)

[10] Y.S. Kim and M.E. Noz 1991 Phase Space Picture of Quantum Mechanics (World Scientific, Singapure)

[11] M.V.Berry 1977 Philos. Trans. R. Soc. London Ser. A 287, 237

[12] N. Mukunda 1979 Am. J. Phys. 47, 182

[13] J. P. Bizarro 1994 Phys. Rev. A 49, 3255

[14] D. Galetti and A. F. R. de Toledo Piza 1988 Physica 149A 267

[15] D. Galetti and A. F. R. de Toledo Piza 1992 Physica A186 513

[16] M. Ruzzi and D. Galetti 1999 J. of Phys. A: Math and Gen. 331065

[17] L. Barker 2001 J. funct. Anal 186153

[18] L. Barker 2001 J. Phys A: Math. and Gen. 224673

[19] L. Barker 2001 J. Math. Phys. 42 (2001) 4653

[20] J. Schwinger 1960 Proc. Nat. Acad. Sci. 46 570,893,1401; 1961 Proc. Nat. Acad. Sci. 471075

[21] D. Galetti and M. A. Marchiolli 1996 Ann. of Phys. 249454

[22] D. Galetti and M. Ruzzi 1999 Physica A 264473

[23] W.K. Wooters 1987 Ann. of Phys.176 1

[24] O. Cohendet, Ph. Combe, M. Siruge and M. Siruge Collin 1988 J. Phys A: Math. and Gen. 212875 